\title{
Fedratinib Hydrochloride
}

National Cancer Institute

\section{Source}

National Cancer Institute. Fedratinib Hydrochloride. NCI Thesaurus. Code C162995.

The monohydrate dihydrochloride salt form of fedratinib, an orally bioavailable, smallmolecule, ATP-competitive inhibitor of Janus-associated kinase 2 (JAK2) and FMS-like tyrosine kinase 3 (FLT 3; CD135; ST K1; FLK2), with potential antineoplastic activity. Upon oral administration, fedratinib competes with wild-type JAK2 as well as mutated forms for ATP binding, which may result in inhibition of JAK2 activation, inhibition of the JAK-STAT signaling pathway, inhibition of tumor cell proliferation and induction of tumor cell apoptosis. JAK2 is the most commonly mutated gene in bcr-abl-negative myeloproliferative disorders (MPDs). In addition, fedratinib targets, binds to and inhibits the activity of FLT3. This inhibits uncontrolled FLT3 signaling and results in the inhibition of proliferation in tumor cells overexpressing FLT3. FLT3, a class III receptor tyrosine kinase (RTK), is overexpressed or mutated in most B-lineage neoplasms and in acute myeloid leukemias and plays a key role in tumor cell proliferation. 